Tai Xi-Shi*, Liu Li-Li and Zhou Xiao-Jing

\title{
The crystal structure of poly[ $\mathrm{m}_{2}$-aqua-tetraaqua- bis( $m_{9}$-4-formylbenzene-1,3-disulfonato) tetrasodium(I) hydrate, $\mathrm{C}_{14} \mathrm{H}_{18} \mathrm{O}_{19} \mathrm{~S}_{4} \mathrm{Na}_{4}$
}

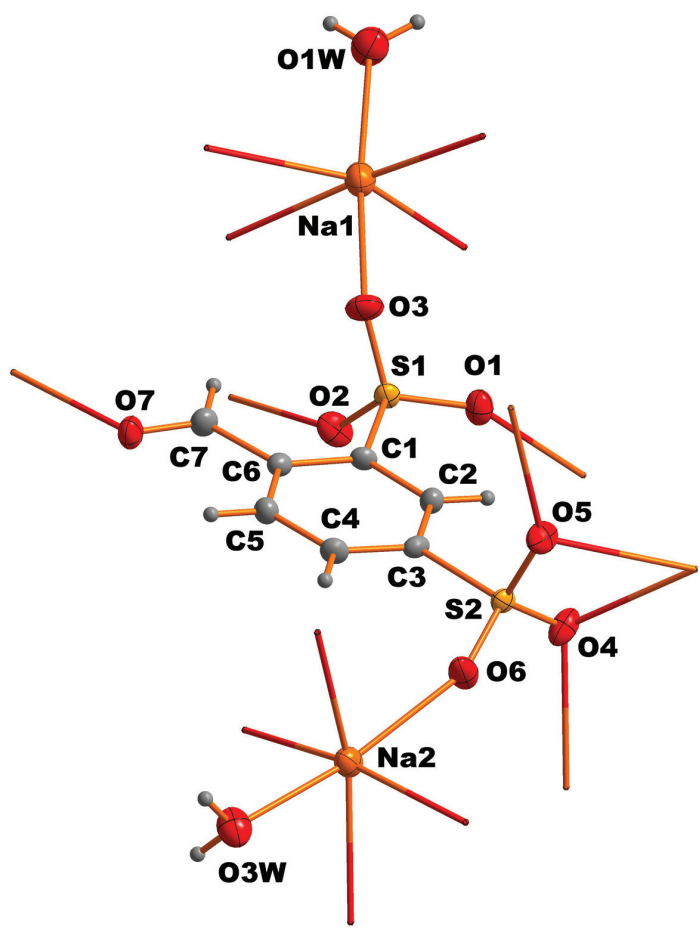

https://doi.org/10.1515/ncrs-2019-0035

Received January 12, 2019; accepted March 5, 2019; available online March 26, 2019

\section{Abstract \\ $\mathrm{C}_{14} \mathrm{H}_{18} \mathrm{O}_{19} \mathrm{~S}_{4} \mathrm{Na}_{4}$, triclinic, $P \overline{1} \quad$ (no. 2), $\quad a=7.0769(14) \AA$, $b=9.5622(19) \AA, \quad \quad c=10.952(2)) \AA, \quad \alpha=115.14(3)^{\circ}$, $\beta=90.46(3)^{\circ}, \quad \gamma=106.53(3)^{\circ}, \quad V=636.1(2) \AA^{3}, \quad Z=1$, $R_{\mathrm{gt}}(F)=0.0448, w R_{\text {ref }}\left(F^{2}\right)=0.1191, T=293(2) \mathrm{K}$.}

\section{CCDC no.: 1901183}

The molecular structure is shown in the figure. Table 1 contains crystallographic data and Table 2 contains the list

*Corresponding author: Tai Xi-Shi, College of Chemistry and Chemical Engineering, Weifang University, Weifang, Shandong 261061, P.R. China, e-mail: taixs@wfu.edu.cn Liu Li-Li and Zhou Xiao-Jing: College of Chemistry and Chemical Engineering, Weifang University, Weifang, Shandong 261061, P.R. China
Table 1: Data collection and handling.

\begin{tabular}{ll}
\hline Crystal: & Yellow block \\
Size: & $0.21 \times 0.20 \times 0.19 \mathrm{~mm}$ \\
Wavelength: & Mo $K \alpha$ radiation $(0.71073 \AA)$ \\
$\mu:$ & $0.53 \mathrm{~mm}^{-1}$ \\
Diffractometer, scan mode: & Bruker, $\varphi$ and $\omega$ \\
$\theta_{\text {max }}$, completeness: & $25.0^{\circ},>99 \%$ \\
$N\left(h k l_{\text {measured }}, N\left(h k l_{\text {unique }}, R_{\text {int }}:\right.\right.$ & $4997,2246,0.040$ \\
Criterion for $I_{\text {obs }}, N\left(h k l l_{\text {gt }}:\right.$ & $I_{\text {obs }}>2 \sigma\left(I_{\text {obs }}\right), 2128$ \\
$N(\text { param })_{\text {refined }}:$ & 185 \\
Programs: & Bruker [1], SHELX $[2,3]$, \\
& Diamond [4] \\
\hline
\end{tabular}

Table 2: Fractional atomic coordinates and isotropic or equivalent isotropic displacement parameters $\left(\AA^{2}\right)$.

\begin{tabular}{lrrrr}
\hline Atom & $\boldsymbol{x}$ & $\boldsymbol{y}$ & $\boldsymbol{z}$ & $\boldsymbol{U}_{\text {iso }}{ }^{*} \boldsymbol{U}_{\text {eq }}$ \\
\hline S1 & $-0.07669(8)$ & $-0.68903(6)$ & $0.29435(5)$ & $0.0184(2)$ \\
S2 & $-0.24073(8)$ & $-1.33612(6)$ & $0.16300(6)$ & $0.0183(2)$ \\
Na1 & $-0.25680(16)$ & $-0.35031(14)$ & $0.43253(11)$ & $0.0348(3)$ \\
Na2 & $-0.22474(15)$ & $-1.62759(12)$ & $-0.19927(10)$ & $0.0272(3)$ \\
C1 & $-0.1633(3)$ & $-0.8975(3)$ & $0.1675(2)$ & $0.0177(5)$ \\
C2 & $-0.1629(3)$ & $-1.0170(3)$ & $0.2079(2)$ & $0.0189(5)$ \\
H2A & -0.1132 & -0.9882 & 0.2971 & $0.023^{*}$ \\
C3 & $-0.2380(3)$ & $-1.1805(3)$ & $0.1132(2)$ & $0.0176(5)$ \\
C4 & $-0.3142(3)$ & $-1.2257(3)$ & $-0.0199(2)$ & $0.0205(5)$ \\
H4A & -0.3679 & -1.3352 & -0.0814 & $0.025^{*}$ \\
C5 & $-0.3094(3)$ & $-1.1060(3)$ & $-0.0602(2)$ & $0.0204(5)$ \\
H5A & -0.3574 & -1.1357 & -0.1500 & $0.024^{*}$ \\
C6 & $-0.2332(3)$ & $-0.9409(3)$ & $0.0321(2)$ & $0.0182(5)$ \\
C7 & $-0.2353(4)$ & $-0.8193(3)$ & $-0.0177(2)$ & $0.0237(5)$ \\
H7A & -0.2005 & -0.7103 & 0.0457 & $0.028^{*}$ \\
O1 & $-0.0144(3)$ & $-0.6955(2)$ & $0.41816(17)$ & $0.0285(4)$ \\
O1W & $-0.2952(3)$ & $-0.0887(3)$ & $0.5752(2)$ & $0.0432(5)$ \\
H1WB & -0.2919 & -0.0486 & 0.6619 & $0.065^{*}$ \\
H1WA & -0.2786 & -0.0083 & 0.5559 & $0.065^{*}$ \\
O2 & $0.0871(3)$ & $-0.6030(2)$ & $0.24795(19)$ & $0.0320(5)$ \\
O2W & $-0.5558(8)$ & $-0.5381(7)$ & $0.4310(5)$ & $0.0584(13)^{*}$ \\
H2WA & -0.5690 & -0.4527 & 0.4879 & $0.088^{*}$ \\
03 & $-0.2486(3)$ & $-0.6335(2)$ & $0.30245(19)$ & $0.0319(5)$ \\
O3W & $-0.3076(3)$ & $-1.8041(3)$ & $-0.4411(2)$ & $0.0393(5)$ \\
H3WA & -0.2192 & -1.7738 & -0.4860 & $0.059^{*}$ \\
H3WB & -0.4228 & -1.8298 & -0.4858 & $0.059^{*}$ \\
04 & $-0.0710(3)$ & $-1.2698(2)$ & $0.2689(2)$ & $0.0321(4)$ \\
05 & $-0.4261(3)$ & $-1.3706(2)$ & $0.21709(19)$ & $0.0291(4)$ \\
06 & $-0.2264(4)$ & $-1.4712(2)$ & $0.0404(2)$ & $0.0401(5)$ \\
07 & $-0.2797(3)$ & $-0.8543(2)$ & $-0.13607(17)$ & $0.0288(4)$ \\
\hline 0cCupancy & 0.5. & & &
\end{tabular}

accupancy: 0.5 . 
of the atoms including atomic coordinates and displacement parameters.

\section{Source of material}

Disodium 4-formylbenzene-1,3-disulfonate (0.1551 g; $0.5 \mathrm{mmol}$ ) was dissolved in $3 \mathrm{~mL} \mathrm{H}_{2} \mathrm{O}$ at room temperature. An amount of $10 \mathrm{~mL} \mathrm{CH}_{3} \mathrm{CH}_{2} \mathrm{OH}$ containing $0.0685 \mathrm{~g}$ 3-pyridinecarboxylic hydrazide $(0.5 \mathrm{mmol})$ was added with stirring. The mixture was stirred for $6.5 \mathrm{~h}$ at $75^{\circ} \mathrm{C}$. The crystals of the title compound were obtained by slow evaporating the filtrate at room temperature after 15 days.

\section{Experimental details}

The hydrogen atoms were positioned geometrically ( $\mathrm{C}$ $\mathrm{H}=0.93 \AA$ and $\mathrm{O}-\mathrm{H}=0.82-0.86 \AA$ ). Their $U_{\text {iso }}$ values were set to $1.2 U_{\text {eq }}$ or $1.5 U_{\text {eq }}$ of the parent atoms. One water molecule is only half occupied.

\section{Comment}

Studies on coordination polymers with various structures are of current interest, because they have shown potential applications in many fields such as catalysis, luminecence, antitumor and antibacterial activities [5-7]. In order to explore the synthesis, structure and property of coordination polymers a new coordination polymer was synthesized and structurally characterized.

In the title compound, each $\mathrm{Na}(\mathrm{I})$ atom is six-coordinated with four $\mathrm{O}$ atoms of four different $\mathrm{SO}_{3}{ }^{-}$groups from four different benzaldehyde disulfonate ligands, one $\mathrm{O}$ atom of aldehyde group and one $\mathrm{O}$ atom from coordinated water molecule, which forms a distorted octahedral geometry.
The title compound forms a three-dimensional network structure by the bridging effect of the organic disulfonato ligand. As shown in the figure each disulfonato ligand coordinates to nine crystallographically related, neighboring sodium cations.

Acknowledgements: This project was supported by the National Natural Science Foundation of China (no. 21171132), the Natural Science Foundation of Shandong (ZR2014BL003), the project of Shandong Province Higher Educational Science and Technology Program (J14LC01) and Science Foundation of Weifang.

\section{References}

1. Bruker. SAINT and SADABS. Bruker AXS Inc., Madison, WI, USA (2000).

2. Sheldrick, G. M.: A short history of SHELX. Acta Crystallogr. A64 (2008) 112-122.

3. Sheldrick, G. M.: Crystal structure refinement with SHELXTL. Acta Crystallogr. C71 (2015) 3-8.

4. Brandenburg, K.: DIAMOND. Visual Crystal Structure Information System. Ver. 3.2. Crystal Impact, Bonn, Germany (2012).

5. Tai, X.-S.; Wang, X.; Li, P.-F.: Synthesis, crystal structure, and luminescent property of a Cd(II) coordination polymer with a $\mathrm{N}$-nicotinoylglycine ligand. Crystals 7 (2017) 33.

6. Song, T.-Q.; Dong, J.; Gao, H.-L.; Cui, J.-Z.: Three coordination polymers based on $M_{2}(M=C o, N i$ and $Z n)$ clusters: structures, magnetic and fluorescent properties. Inorg. Chim. Acta 466 (2017) 393-397.

7. Tai, X.-S.; Wang, X.: Synthesis, structural characterization and antitumor activity of a Ca(II) coordination polymer based on 4formyl-1,3-benzenedisulfonate-2-furoic acid hydrazide ligand. Crystallogr. Rep. 62 (2017) 242-245. 